\title{
Cartografía de Unidades Ambientales Homogéneas (UAH): Un insumo para el ordenamiento del bosque chaqueño en Salta
}

\author{
María C. Camardelli ${ }^{1, \otimes}$; Santiago Miranda ${ }^{1}$ \& Gisela S. Córdoba ${ }^{2}$ \\ ${ }^{1}$ Universidad Nacional de Salta. ${ }^{2}$ Instituto de Bio y Geociencias del NOA-CONICET.
}

\begin{abstract}
Resumen. En la provincia de Salta, la Ley 7543 de Ordenamiento Territorial del Bosque Nativo zonificó el territorio al norte del río Bermejo dentro de las categorías I y II, esta última destinada al aprovechamiento forestal y a sistemas silvopastoriles (SSP) o a ganadería bajo monte. Las tecnologías para implantar pasturas en SSP se adecuan a la Ley y se están implementando exitosamente en sitios con bosque alto en suelos de fuerte desarrollo, pero no en arbustales con baja cobertura vegetal, donde se pierden rápidamente. El objetivo de este trabajo es elaborar cartografía de las Unidades Ambientales Homogéneas (UAH) que cubren el Sitio Piloto Chaco Semiárido (SPChSA) de la red del Observatorio Nacional de Degradación de Tierras y Desertificación (ONDTyD), agrupadas por criterios de vegetación y suelos como insumo para el ordenamiento del uso del territorio. Mediante un sistema de información geográfica (SIG) y muestreos de suelos y vegetación se efectuó una clasificación supervisada que arrojó seis UAH. Se elaboró cartografía temática del sitio piloto en la que se muestra, a escala de semidetalle, la distribución de las UAH y la superficie que ocupa cada UAH. Se encontró que 30\% del área Categoría II está cubierta con arbustales que no poseen aptitud para mantener SSP sostenibles en el largo plazo, pero que fueron descritos como valiosos para el funcionamiento del ecosistema. Esto constituye un alerta sobre la necesidad de mapear de forma apropiada las áreas que deben ser resguardadas en la zonificación vigente, a fin de no profundizar procesos de degradación en el territorio.
\end{abstract}

[Palabras clave: Ley 7543 OTBN Salta, caracterización de la vegetación, clasificación textural de suelos, SIG, zonificación del territorio]

\begin{abstract}
Aвstract. Cartography of Homogeneous Environmental Units in Salta: Input for ordering the Chaco forests. The north of the Bermejo River, territory of Salta province, was zoned as categories I and II, according to the Law 7543 of Native Forest Territorial Planning. The Category II allows forest use or raising cattle under forests. The technologies involved in implanting pastures under silvopastoral systems meet the requirements of the Law and are being successfully implemented in sites with high forest and developed soils, but not in shrubland with low vegetation cover, where they quickly disappear. The aim of this work is to elaborate cartography of the Homogeneous Environmental Units (HEU) covering Semiarid Chaco Pilot Site (SPChSA) of the National Observatory of Land Degradation and Desertification (ONDTyD), grouped by criteria of vegetation and soils, as input for land use planning. A supervised classification of the study area was carried out using geographic information system (GIS) and sampling soil and vegetation. Six EHU were identified. Thematic mapping of the pilot site was prepared, and the area occupied by each unit was calculated as a contribution to current environmental legislation. It is reported that $30 \%$ of Category II is covered with shrublands that do not have the ability to maintain sustainable SSP in the long term, but are valuable for the functioning of the ecosystem. This is a warning of the need to properly map the areas that should be protected in the current zoning to avoid further degradation processes in the territory.
\end{abstract}

[Keywords: Law 7543 Salta OTBN, characterization of vegetation, soil textural classification, GIS, territory zoning]

\section{INTRODUCCIÓN}

Las Unidades Ambientales Homogéneas (UAH) que dividen un área constituyen recintos con cierta uniformidad interna en cuanto a sus caracteres bióticos y físicos. Se caracterizan por presentar una fisonomía homogénea y una evolución común, con dimensiones concretas y cartografiables (Dorta et al. 2014). La delimitación y representación cartográfica de las UAH contribuye en los procesos de zonificación y ordenamiento de uso del suelo.

Editora asociada: Patricia Kandus
El avance de la frontera agrícola y ganadera en la Argentina supuso un proceso profundo de cambio en el uso del suelo. En ciertas regiones del país (e.g., la provincia de Salta), esto suscitó una tasa inédita de deforestación de bosques nativos (Schmidt 2014), que en el 2007 llevó a sancionar la Ley de Presupuestos Mínimos de Protección Ambiental de los Bosques Nativos en la República Argentina (Ley 26331), y en el 2008 a sancionar la Ley 7543 en la provincia de Salta. A partir de la aplicación de la Ley 7543 en Salta, los bosques al norte del río Bermejo quedaron 
zonificados en su mayor parte en dos de las tres categorías sancionadas: Categoría I (roja), que corresponde a sectores de muy alto valor de conservación que no deben transformarse, y Categoría II (amarilla), de mediano valor de conservación, donde se podrán establecer: “...sistemas de Ganadería Silvo-Pastoril o bajo monte..." (Ley $\mathrm{N}^{\circ} 7543$, Boletín Oficial 18035, enero de 2009). Este mandato buscó limitar el uso de tecnologías de desmontes totales y selectivos tal cual se venían realizando en el territorio, promoviendo la ejecución de sistemas silvopastoriles (SSP) basados en "...el uso de los bosques nativos de forma e intensidad que permita mantener su biodiversidad, productividad, vitalidad, potencialidad y capacidad de regeneración... manteniendo los Servicios Ambientales que prestan a la sociedad" (Art. 4, Ley 26331 Boletín Oficial 31310, diciembre de 2007).

Sin embargo, es necesario mencionar que en la región chaqueña se ha generalizado como SSP una práctica cuyo interés es exclusivamente ganadero. Para implementarla, los ganaderos eliminan totalmente el estrato arbustivo del bosque mediante rolados con maquinaria de gran porte, dejando en pie sólo árboles maduros. Para estos sitios rolados, Guevara et al. (2020) reportan que la mayor disponibilidad de luz y el efecto propio del accionar del rolo estimulan el rebrote basal, lo cual modifica la composición y la arquitectura de las especies arbustivas de las unidades intervenidas. Esto genera la rápida arbustización de los potreros. Para controlar este proceso se aplican rerolados o rastreados, cuya intensidad y frecuencia afectan la persistencia del estrato arbóreo, argumento suficiente para no considerarlos SSP (Despósito and Ledesma 2015).

Los SSP que cumplen con el mandato de la Ley son los que mantienen la composición y la funcionalidad del bosque. Algunos de ellos se instalaron en predios de campesinos criollos de la zona de estudio, y sus principales características fueron informadas por Camardelli et al. (2015). Se caracterizan por una mínima intervención sobre los estratos arbóreos y arbustivos; sólo se podan las ramas bajas o caídas de los arbustos que dificultan la circulación (elementos que en la región se denominan 'champas'), que se reducen y distribuyen sobre la superficie del suelo junto con el material vegetal remanente de árboles o arbustos enfermos o muertos. Una vez deschampado el bosque, se siembra de manera directa una pastura umbrófila. El bosque se mantiene como una unidad funcional, producto de un estrato arbóreo diversificado y un estrato arbustivo en equilibrio, no invasor (Camardelli et al. 2012), con escasa presencia de suelos sin cobertura (Guevara et al. 2020).

Estudios llevados a cabo en predios de campesinos criollos donde se implementaron SSP mediante deschampados informan que las pasturas de Megathyrsus maximus (gatton panic) se comportaron de distinta manera según su ubicación. Las pasturas implantadas bajo unidades de vegetación de bosque alto, en suelos de fuerte desarrollo, francos a franco limosos y contenido de materia orgánica (MO) medio (3\%) produjeron una mayor cantidad de forraje acumulado. Por su parte, las pasturas que produjeron menos fueron las cultivadas en unidades con vegetación de porte bajo o arbustales en suelos con texturas finas arcillo-limosa y contenidos de $\mathrm{MO}$ menores a $2 \%$ (en este último caso, con el agravante de que se perdieron [Camardelli et al. 2005]). La menor producción y persistencia de las pasturas implantadas en los arbustales se atribuye a impedimentos derivados de las características físico-químicas de los suelos (Caruso et al. 2012) y de una estructura vertical de la vegetación arbórea y arbustiva que no contribuye de manera importante con residuos orgánicos al sistema (Camardelli et al. 2005).

Nos proponemos como objetivo indagar la distribución y la superficie que ocupan las UAH agrupadas por criterios de vegetación y clasificación textural de los suelos, y elaborar cartografía a una escala semidetallada como insumo para orientar la ejecución de SSP hacia los sectores con mayores perspectivas de sustentabilidad, resguardando, a la vez, aquellas UAH que se reportaron como no aptas para sostener estas intervenciones, pero que constituyen sectores valiosos por sus funciones ecosistémicas.

\section{Materiales y Métodos}

\section{Ubicación y características del área de estudio}

El área de estudio cubre el Sitio Piloto Chaco Semiárido(SPChSA)delObservatorio Nacional de Degradación de Tierras y Desertificación (ONDTyD), ubicado al norte del río Bermejo, en el sudeste del departamento San Martin y el suroeste del departamento Rivadavia, de la provincia de Salta (23.3423 S ' 63.4318 O). El sitio permanece mayoritariamente cubierto con bosque nativo, y está habitado por comunidades aborígenes y pequeños 
productores criollos, con una baja penetración de agroempresas. Corresponde a uno de los 17 sitios del sistema nacional de evaluación y monitoreo de tierras del ONDTyD, creados con el objetivo proveer información relativa al estado, tendencias y riesgos de la degradación de tierras en la Argentina mediante el monitoreo de indicadores biofísicos y socioeconómicos (Therburg et al. 2019).

Fitogeográficamente, corresponde a la Provincia Chaqueña, Distrito Chaqueño Occidental (Cabrera 1976). Fisonómicamente, es un bosque de xerofitas con Schinopsis lorentzii (Griseb.) Engl. (quebracho colorado) y Aspidosperma quebracho-blanco Schlecht (quebracho blanco) (Cabrera 1976). En los suelos de topografía más elevada, moderadamente bien drenados hasta algo imperfectamente drenados, con texturas desde franco-limosas, franco-arcillosas a areno- limosas y limo-arcillosas, se desarrollan los bosques xéricos propios de las llanuras aluviales. Son bosques bajos con dosel denso de 5 a $7 \mathrm{~m}$ y emergentes dispersos que alcanzan los 15 a $20 \mathrm{~m}$ de altura. En los suelos mal drenados con texturas arcillo-limosas, la vegetación corresponde a bosques bajos y arbustales con dosel denso de 3 a $6 \mathrm{~m}$ y emergentes dispersos de 10 a 16 m (Morello et al. 2012).

\section{Secuencia de actividades realizadas para obtener la cartografía}

1. Leyenda cartográfica a priori. Se elaboró una leyenda a priori de coberturas del suelo en base a consulta de expertos y cartografía temática disponible con leyendas de clasificación basadas en Land Cover Classification System - LCCS. Este método permite elaborar leyendas ad hoc siguiendo criterios jerárquicos preestablecidos: presencia/ausencia de vegetación, condición edáfica (terrestre/anegable-acuática) y tipo de cobertura (natural/artificial). A su vez, estos criterios se subdividen a partir de variables que dependen de cada tipo de cubierta y según la región en estudio, el nivel de detalle y el objetivo del trabajo (Volante et al. 2009) (Caja 2).

2. Clasificación preliminar - NDVI. Se seleccionó una imagen provista por el satélite Landsat 8 con fecha del 6 de octubre de 2015, utilizando la plataforma LandViewer del sitio web EOS (Earth Observing System). La fecha de selección de la imagen corresponde al fin del período seco, con lo que se consigue un producto con menor cantidad de nubes y una mejor visualización de la vegetación (Alencar da Silva Alves et al. 2019). En el procesamiento de la imagen satelital y las capas vectoriales se empleó el software ArcGis 10.0 (software licenciado por IBIGEO CONICET), con una escala de procesamiento de 1:50.000 y una unidad mínima de mapeo de 10 ha.

Para identificar diferencias en los tipos de coberturas se calculó el índice de vegetación normalizado (NDVI), que permite representar el vigor y la cantidad de vegetación a través de su relación con la actividad fotosintética (Chuvieco 1995) y es usado en el proceso de monitoreo del ONDTyD (Gaitán et al. 2013) (Figura 1). El algoritmo correspondiente es: banda5- banda4/banda5+banda4.

Si bien emplear una sola imagen para clasificar la cobertura del suelo por medio del índice NDVInoes una propuesta metodológica usada para identificar la evolución de la vegetación o cambios de uso del suelo de un determinado territorio (Guerschman et al. 2003), en la Región Chaqueña se la utiliza para contrastar la vegetación activa e identificar patrones espaciales de las unidades de vegetación nativa (Volante and Bianchi 2003; Cabral et al. 2009). También se la usó en el Plan de Ordenamiento Territorial para las Áreas Boscosas de la Provincia de Salta anexo del Decreto 2785/09, que produjo la zonificación de base del Ordenamiento Territorial de Bosque Nativo en esta provincia (Boletín Oficial Salta No 18144, 13/07/09).

3. Selección de los puntos de control (PC). Con el objeto de identificar zonas representativas (Araya Morales 2009), se emplazaron y georreferenciaron PC a campo sobre las coberturas de vegetación identificadas a priori. El cálculo del número de PC necesarios para obtener un error de muestreo tolerable se realizó mediante la siguiente fórmula:

$$
\mathrm{n}=\left(\mathrm{N} \cdot \sigma^{2} \cdot \mathrm{Z}^{2}\right) /\left[(\mathrm{N}-1) \cdot \mathrm{e}^{2}+\left(\sigma^{2} \cdot \mathrm{Z}^{2}\right)\right]
$$

donde $\mathrm{n}=$ número de $\mathrm{PC}$; $\mathrm{N}$ =total de píxeles; e=error (99.9 de precisión); $\sigma=$ desvío estándar; $\mathrm{Z}=$ margen de confiabilidad (95\%) (García 2004).

4. Muestreo a campodelos PC, caracterización de la vegetación y clases texturales de suelos. Definición de umbrales. La caracterización de la vegetación formó parte del trabajo de monitoreo de indicadores biofísicos que se 
lleva a cabo en el SPChSA en el marco del ONDTyD desertificacion.gob.ar/sitiospiloto). El relevamiento de la vegetación leñosa (i.e., árboles y arbustos) se efectuó en 40 parcelas de 100x20 m. Para separar estas dos formas de vida se registraron como árboles las plantas con un solo tronco leñoso, capaces de alcanzar al menos una altura de $5 \mathrm{~m}$ en la madurez. En tanto, las plantas leñosas perennes con más de un vástago principal y sin una copa definida fueron clasificadas como arbustos (FAO 2020). En cada parcela $\left(2000 \mathrm{~m}^{2}\right)$ se registró cobertura y frecuencia de especies leñosas según la metodológica del ONDTyD (desertificación.gob.ar/indicadores/ vegetación). a) Cobertura: sobre la línea central longitudinal de cada parcela, y cada $10 \mathrm{~m}$, se estimó de forma visual la proyección de copa de la vegetación leñosa sobre el terreno. Se registró, además, la presencia de suelo sin cobertura vegetal viva o muerta (\%) (suelo desnudo). b) Frecuencia: se contaron todos los árboles presentes y se registró a qué especie pertenecía cada uno. En cuanto al estrato arbustivo, se registraron las especies más conspicuas siguiendo un orden de frecuencia de aparición decreciente.

En el caso de las tierras desmontadas, se registró el tipo de cultivo presente. Para caracterizar los tipos fisonómico-florísticos, la metodología propone dos criterios. 1) Vegetación cerrada (cobertura vegetal total mayor a $75 \%$ ), abierta (cobertura vegetal total entre 25 y $75 \%$ ) o rala (cobertura vegetal total menor al 25\%). 2) Forma de vida dominante árboles, arbustos, o gramíneas. Para ajustar umbrales de cobertura se usaron los datos de densidad de árboles expresada en número de ejemplares por ha.
Caja 1. Flujograma de actividades para identificar UAH y elaborar cartografía temática del SPChSA.

Box 1. Flowchart of activities to identify UAH and develop thematic mapping of the SPChSA

1. Construcción de la leyenda cartográfica a priori

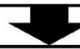

2. Clasificación preliminar - NDVI

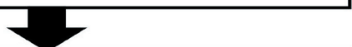

3. Selección y georreferenciación de los puntos de control (PC)

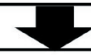

4. Muestreo de campo. Definición de umbrales

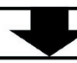

5. Clasificación Supervisada con detalle de UAH con criterios de vegetación y suelos

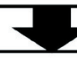

6. Validación de los resultados

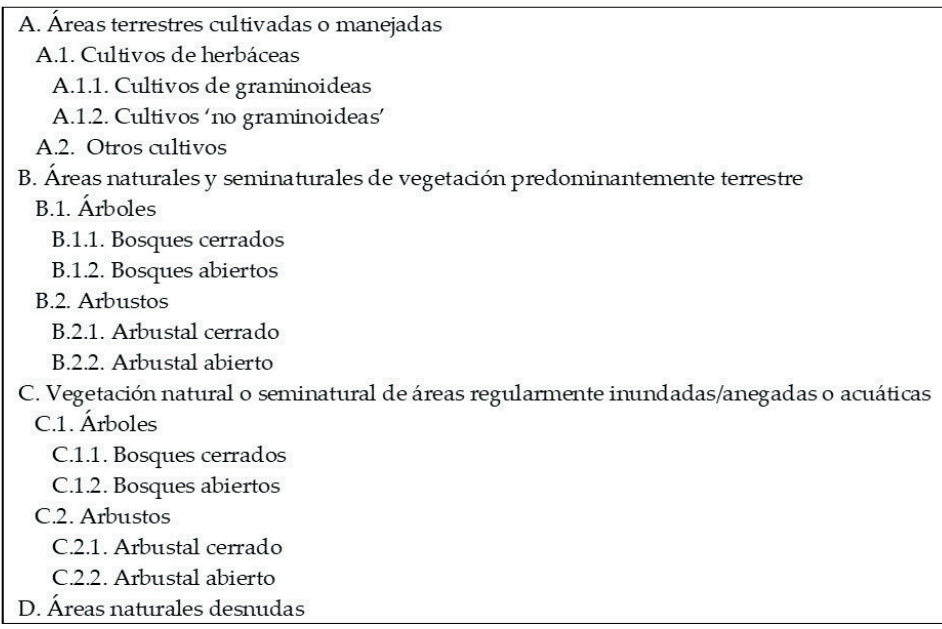

Caja 2. Leyenda cartográfica $a$ priori de las coberturas del suelo presentes en el SPChSA (adaptada de Volante et al. 2009).

Box 2. A prori cartographic legend of the land covers present in the SPChSA (adapted from Volante et al. 2009). 
Para conocer la textura de los suelos se tomaron muestras compuestas (formadas por tres submuestras) de los primeros $0.3 \mathrm{~m}$. Las muestras se tomaron en el área central de cada uno de los PC y se determinó textura mediante el método del hidrómetro de Bouyoucos (1962).
5. Clasificación final y construcción de la leyenda de UAH. Con la información de cada PC y los umbrales descriptos anteriormente se elaboraron tablas relacionando las variables de cobertura, formas de vida y textura de los suelos. Esto se asoció con las leyendas a priori de cobertura de vegetación, procediendo,

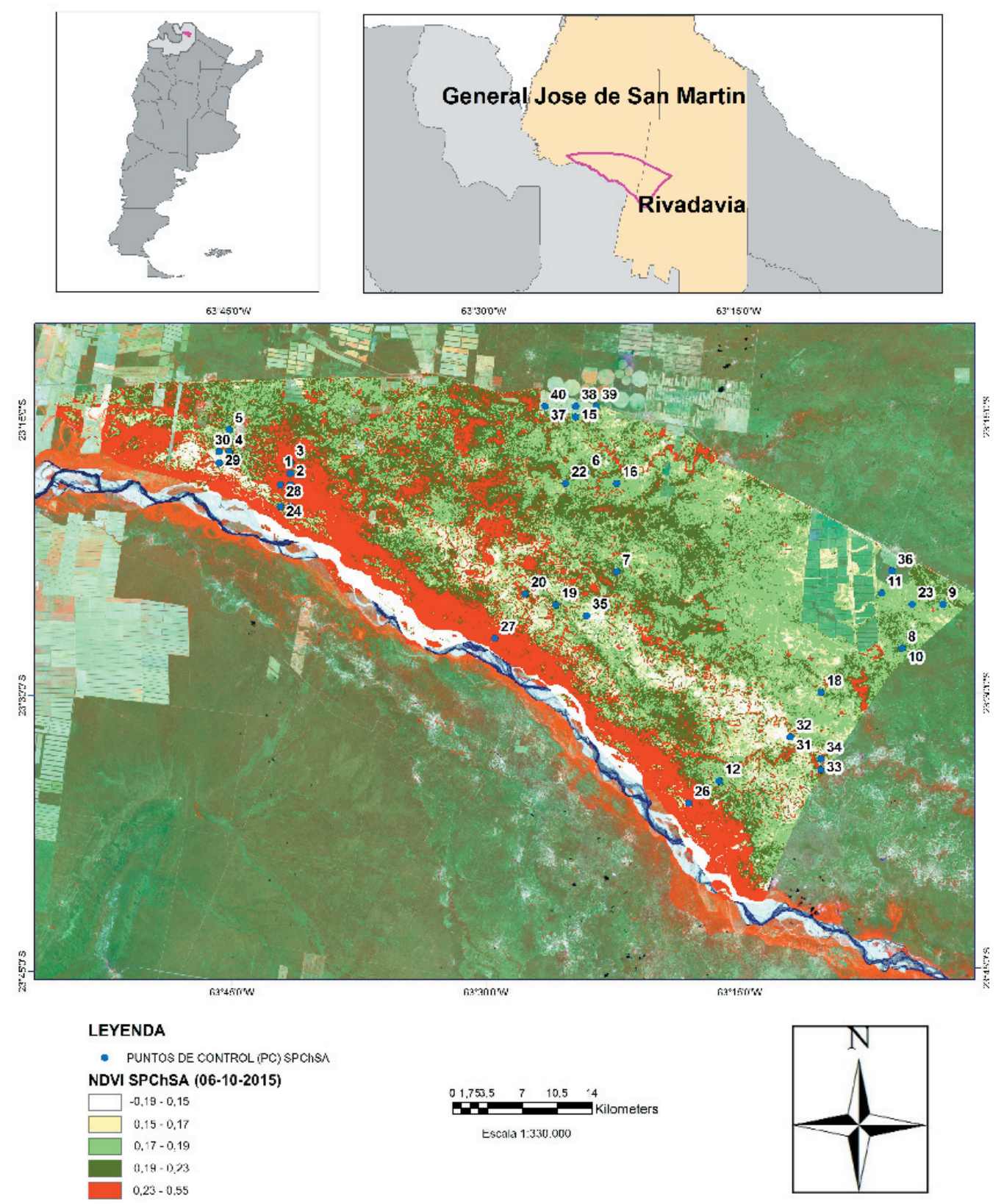

Figura 1. Área de estudio y recorte del SPChSA donde se muestra el índice de vegetación normalizado (NDVI) clasificado de manera preliminar. Se observan los patrones espaciales de la distribución de cada clase, sobre los que se emplazaron y georreferenciaron los puntos de control (PC) (círculos numerados) usados para capturar las clases de entrenamiento en la clasificación final.

Figure 1. Study area and SPChSA clipped showing the normalized vegetation index (NDVI) preliminarily classified. The spatial patterns of the distribution of each class are shown, on which the control points (PC) (numbered circles) used to capture the training classes in the final classification were placed and georeferencing. 
cuando correspondía, a ajustes de esas leyendas.

Se realizó una clasificación supervisada basada en áreas de entrenamiento construidas sobre la imagen raster del área de estudio. Se digitalizaron polígonos en base a los puntos de control de campo (40 PC), que recogen las respuestas espectrales de las unidades de vegetación identificadas a priori. La clasificación supervisada se realizó empleando el algoritmo de máxima probabilidad, basado en un algoritmo paramétrico que cuantifica la probabilidad de que un píxel pertenezca a una clase particular, asignando el píxel a aquella que maximice la función de probabilidad (Guevara et al. 2020). La imagen final resultante de esta clasificación fue recodificada a las clases correspondientes de la leyenda de UAH identificada. Se vectorizó el producto raster final obtenido, se agruparon los polígonos pertenecientes a una misma clase de UAH y se le agregó la leyenda final teniendo en cuenta el tipo de cobertura y la clasificación textural (Caja 1).

6. Validación de la clasificación. Se evalúo la confiabilidad de la clasificación a través de una matriz de confusión, en la que se contrastó la verdad de terreno con la información del mapa clasificado. En esta matriz, las filas se ocupan por las clases de referencia y las columnas se ocupan con las categorías deducidas de la clasificación (Chuvieco 1995). El interés por construir una matriz de confusión deriva de su capacidad para plasmar conflictos entre categorías. Se seleccionaron 50 puntos de referencia por cada $\mathrm{UAH}$, que no coincidían con los PC usados como áreas de entrenamiento. La selección se realizó a partir de la visualización de imágenes de alta resolución espacial (ARS) tomadas de la iniciativa Collect Earth Online, de conocimiento experto y de campañas a terreno. Además de la exactitud global, se calculó el índice de Kappa. Este índice utiliza las sumas marginales de la matriz y referencia la contribución del azar en la confiabilidad del mapa (Mas et al. 2003).

\section{Resultados}

Como producto de la clasificación se obtuvieron 6 categorías a nivel de tipo de cobertura, cuyas leyendas se complementaron con la clase textural de suelos. Cuatro clases corresponden a áreas naturales o seminaturales de vegetación predominantemente terrestre, una clase de vegetación natural o seminatural de áreas regularmente inundadas/anegadas o acuáticas, y una clase de áreas terrestres cultivadas o manejadas, sobre suelos de clases texturales contrastantes (Tabla 1 y Figura 2).

El Bosque Cerrado de ribera cubre las márgenes del río Bermejo y también los albardones de las cañadas y arroyos. Según los censos realizados en el estrato arbóreo, las especies más conspicuas son Prosopis

Tabla 1. Superficie por tipo de cobertura y valores promedio de los indicadores de vegetación registrados (cobertura vegetal total y densidad arbórea) en cada una de las clases de cobertura, discriminada según la nomenclatura de Land Cover Classification System - LCCS. En la última columna se detalla la clasificación textural de los suelos donde se asienta cada cobertura y las texturas encontradas por clase se detallan al pie de la tabla.

Table 1. Area by type and average values of the vegetation indicators recorded (total vegetation cover and tree density) in each of the cover classes, discriminated according to the Land Cover Classification System - LCCS nomenclature. The last column details the textural classification of the soils where each one sits. Textures found by class are detailed at the bottom of the table.

\begin{tabular}{|c|c|c|c|c|c|c|}
\hline \multirow[b]{2}{*}{ Tipos de cobertura } & \multicolumn{3}{|c|}{ Superficie } & \multicolumn{2}{|c|}{ Vegetación } & \multirow{2}{*}{$\begin{array}{l}\text { Suelos } \\
\text { Clase } \\
\text { textural }\end{array}$} \\
\hline & $\mathrm{n}$ & ha & $\%$ & $\begin{array}{c}\text { Cobertura } \\
\text { vegetal total } \\
(\%)\end{array}$ & $\begin{array}{l}\text { Densidad } \\
\text { arbórea } \\
(\mathrm{n} / \mathrm{ha})\end{array}$ & \\
\hline Bosque Cerrado de Ribera & 6 & 41029 & 20 & $88.33 \pm 4.08$ & $109.17 \pm 16.25$ & Media \\
\hline Bosque Cerrado & 8 & 65290 & 32 & $88.75 \pm 3.54$ & $128.13 \pm 30.58$ & Media \\
\hline Bosque Abierto & 8 & 24336 & 12 & $80.63 \pm 6.78$ & $62.50 \pm 11.65$ & Media \\
\hline Arbustal Cerrado & 7 & 45681 & 22 & $61.43 \pm 9.00$ & $28.57 \pm 5.56$ & Fina-Gruesa \\
\hline $\begin{array}{l}\text { Arbustal Abierto/Áreas desnudas en sectores } \\
\text { regularmente anegados }\end{array}$ & 6 & 17174 & 8 & $25 \pm 8.37$ & $34.17 \pm 7.36$ & Fina \\
\hline Cultivos & 5 & 1746 & 6 & $26 \pm 8.94$ & ---- & Media \\
\hline
\end{tabular}

Valores promedio \pm error estándar

Gruesa: A, FA; Media: F, FL, FY; Fina: Y, YL 


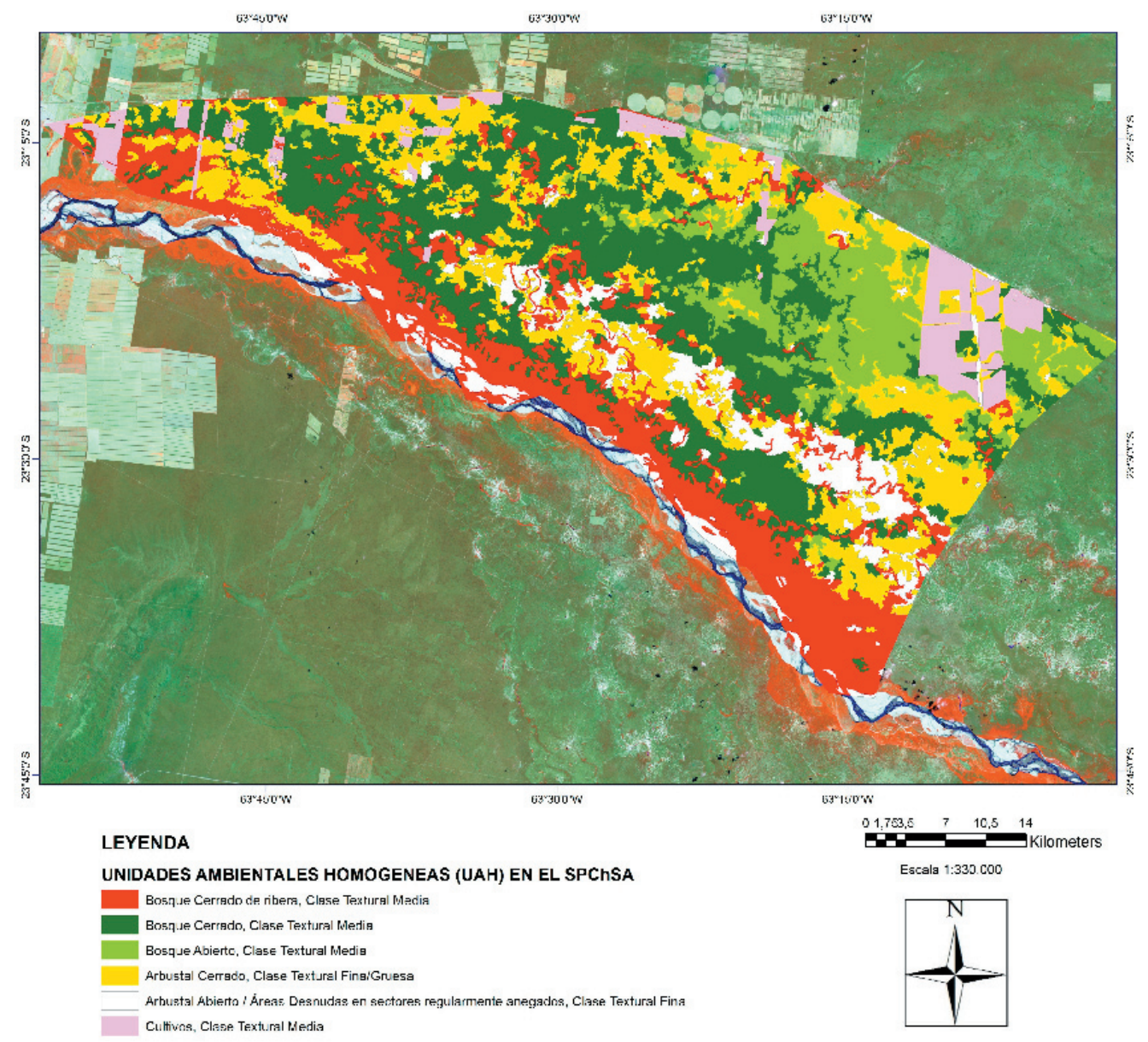

Figura 2. Patrón de distribución de las Unidades Ambientales Homogéneas (UAH), agrupadas por criterios de cobertura de la vegetación y clasificación textural de los suelos. La leyenda sigue la clasificación Land Cover Classification System - LCCS.

Figure 2. Distribution pattern of Homogeneous Environmental Units (UAH), grouped by criteria of vegetation cover and textural classification of soils. Using Land Cover Classification System - LCCS.

alba Griseb. var. alba (algarrobo blanco), Libidibia paraguariensis (D. Parodi) G.P. Lewis (guayacán). Hacia el oeste dominan especies de gran porte Chloroleucon tenuiflorum (Benth.) Barneby and J. W. Grimes (espinillo o tatané) y Geoffroea decorticans (Gillies ex Hook. and Arn.) Burkart (chañar). El muestreo de los suelos en estos PC mostró una fuerte relación con suelos de textura media.

Los Bosques Cerrados y Bosques Abiertos presentan dominancia de quebracho blanco y quebracho colorado, acompañados de Sarcomphalus mistol (Griseb.) Hauenschild (mistol), guayacán, algarrobo blanco, Prosopis nigra (Griseb.) Hieron. var. nigra (algarrobo negro), Gonopterodendron sarmientoi (Lorentz ex Griseb.) A. C. Godoy-Bürki (palo santo). El estrato arbustivo es dominado por Salta triflora (Griseb.) Adr. Sánchez (duraznillo) de hasta $3 \mathrm{~m}$ de altura, seguido por Castela coccinea Griseb. (mistol de zorro o meloncillo) y Anisocapparis speciosa (Griseb.) X. Cornejo and $\mathrm{H}$. H. Iltis (bola verde). Al igual que en la clase anteriormente descrita, el muestreo de estos PC se relacionó con suelos de textura media.

En la cobertura Arbustal Cerrado, bajo el mismo espectro, se encontraron 2 unidades cuyas variables de discriminación fueron: especies arbustivas dominantes y clasificación textural de los suelos. Una de estas unidades se caracteriza por asentarse sobre suelos de textura fina, presenta un estrato arbustivo denso dominado por duraznillo de porte bajo (2 $\mathrm{m}$ de altura) acompañado de Mimozyganthus carinatus (Griseb.) Burkart (iscayante) y Tabebuia nodosa (Griseb.) Griseb. (palo cruz) Emergen algunos árboles como palo santo, quebracho blanco, algarrobo blanco, algarrobo 
negro y mistol. La otra unidad de Arbustal Cerrado identificada es conocida en la región como pichanal o poleal; se asienta sobre suelos franco arenosos a arenosos siguiendo el diseño de los cauces abandonados, domina un estrato subarbustivo compuesto de Senna aphylla (Cav.) H. S. Irwin and Barneby var. Aphylla (pichana o retama) y Lippia sp. (poleo). Emergen ejemplares de quebracho blanco.

Por su parte, el Arbustal Abierto aparece formando agrupaciones de arbustos espinosos sobre áreas desnudas sobre suelos predominantemente finos, con dominancia de Parkinsonia praecox (Ruiz and Pav. ex Hook.) Hawkins (brea), acompañada de Vachellia aroma (Gillies ex Hook. and Arn.) Seigler and Ebinger (tusca), izcayate, Senegalia praecox (Griseb.) Seigler and Ebinger (garabato) y cactáceas. Entre los árboles emergentes predominan palo santo, algarrobo blanco y algarrobo negro. Unidad descrita como 'peladares del Bermejo' por Morello (1968) (en Firpo Lacoste 2018).

En cuanto a la superficie ocupada por las UAH discriminadas, se estimó que la mayor superficie del SPChSA (64\%) está cubierta con Bosque Cerrado y Bosque Abierto (Tabla 1). Por su parte, los Arbustales Cerrados y Arbustales Abiertos suman 30\% del área, y los Cultivos representan la menor ocupación $(6 \%)$.

\section{Validación del mapa: Matriz de confusión e indice Kappa}

Como se observa en la Tabla 2, la clasificación supervisada asignó correctamente 294 de los 312 puntos muestreados, con un índice Kappa de $93.15 \%$ (exactitud global: $94.23 \%$ ) y una alta coincidencia entre las dos fuentes de información a nivel general y resultados poco atribuibles al azar.

\section{Discusión y CONSIDERACIONES FINALES}

El OTBN categorizó de manera uniforme una zona extensa del SPChSA como Categoría II (amarilla) (Figura 3). En cambio, los resultados muestran que ese sector amarillo no corresponde a una sola UAH, sino a cinco, con características particulares en cuanto a cobertura vegetal y clases texturales de los suelos. De esto se desprende que habrá sectores con aptitudes diferentes para sustentar las prácticas que se implementen. Esto permite inferir que las UAH identificadas como Bosques Cerrados y Bosques Abiertos Clase Textural Media presentan aptitud para instalar SSP que emplean la tecnología del deschampado u otras de similares características aprobadas por Ley, y por ello es pertinente su inclusión en la Categoría II (amarilla).

Por su parte, no es posible validar la inclusión en esta categoría de los sectores cubiertos con Arbustales Cerrados Clase Textural Fina o Gruesa y Arbustal Abierto Clase Textural Fina en áreas desnudas regularmente anegadas, ya que por sus características particulares no presentan aptitud para ofrecer sustentabilidad a largo plazo (Camardelli et al. 2005). Esta es una demanda explícita del Criterio 8 de la Ley 26331 respecto al Potencial de

Tabla 2. Matriz de confusión mostrando el número de observaciones clasificadas y verificadas a campo de: Cultivos textura media (C); Arbustal Abierto/Suelos Desnudos en áreas regularmente anegadas textura fina (ASD), Arbustal Cerrado textura fina/gruesa (AC), Bosque Abierto textura media (BA), Bosque Cerrado textura media (BC), Bosque Cerrado de Ribera textura media (BR), con la Exactitud del Usuario (ExU), Exactitud del productor (ExP), el Error de Comisión (ErrC) y Error de Omisión (ErrO).

Table 2. Confusion matrix showing the number of classified and field-verified observations of Medium texture crops (C); Open Bush/Bare Soils in regularly flooded areas fine texture (ASD), Closed Bush fine/coarse texture (AC), Open Forest medium texture (BA), Closed Forest medium texture (BC), Closed Riparian Forest medium texture (BR), and the User Accuracy, (ExU), Producer Accuracy (ExP), Commission Error (ErrC), Omission Error (ErrO).

\begin{tabular}{|c|c|c|c|c|c|c|c|c|c|c|}
\hline & \multirow[b]{2}{*}{ UAH } & \multicolumn{6}{|c|}{ Datos de la clasificación supervisada } & \multirow[t]{2}{*}{ Total } & \multirow{2}{*}{$\begin{array}{c}\text { ExU } \\
(\%)\end{array}$} & \multirow{2}{*}{$\begin{array}{c}\text { ErrC } \\
(\%)\end{array}$} \\
\hline & & $\mathrm{C}$ & ASD & $\mathrm{AC}$ & BA & $\mathrm{BC}$ & BR & & & \\
\hline \multirow{9}{*}{ 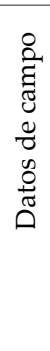 } & C & 52 & 0 & 0 & 0 & 0 & 0 & 52 & 100 & 0 \\
\hline & ASD & 0 & 55 & 0 & 0 & 0 & 0 & 55 & 100 & 0 \\
\hline & $\mathrm{AC}$ & 0 & 0 & 54 & 0 & 0 & 1 & 55 & 98.18 & 1.82 \\
\hline & BA & 0 & 0 & 0 & 48 & 0 & 2 & 50 & 96 & 4 \\
\hline & $\mathrm{BC}$ & 0 & 0 & 3 & 0 & 36 & 11 & 50 & 72 & 28 \\
\hline & BR & 0 & 0 & 0 & 0 & 1 & 49 & 50 & 98 & 2 \\
\hline & Total & 52 & 55 & 57 & 48 & 37 & 63 & 312 & & \\
\hline & $\operatorname{ExP}(\%)$ & 100 & 100 & 94.7 & 100 & 97.2 & 77.7 & & & \\
\hline & ErrO (\%) & 0 & 0 & 5.2 & 0 & 2.7 & 22.2 & & & \\
\hline
\end{tabular}




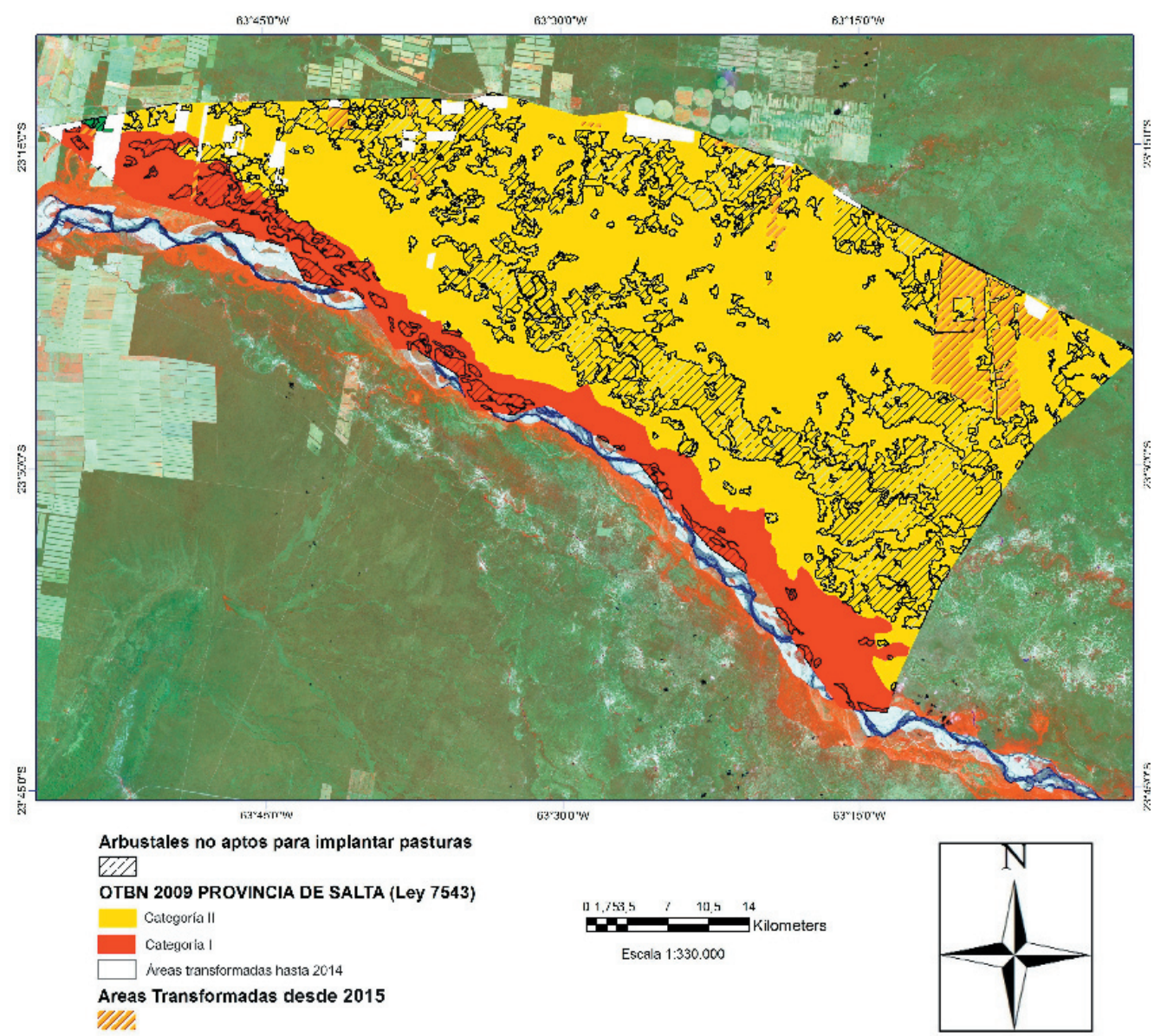

Figura 3. Zonificación de base del Ordenamiento Territorial de Bosque Nativo (OTBN 2009) de la provincia de Salta (Ley 7543) con superposición sobre la Categoría II (amarilla) de las UAH Arbustal Cerrado Clase Textural Fina/Gruesa y Arbustal Abierto/Áreas desnudas en sectores regularmente anegados (barrado diagonal). Los arbustales identificados presentan baja aptitud para un aprovechamiento sustentable, lo que restringe la superficie 'amarilla' aprovechable. El resto de esta zona - con resguardo de los bordes de arroyos y cañadas - correspondería a las UAH Bosque Cerrado y Bosque Abierto Clase Textural Media, en las cuales son mayores las expectativas de sustentabilidad ante intervenciones antrópicas que se pretendan instalar SSP.

Figure 3. Zoning of Native Forests Law (OTBN 2009) (Spanish acronym) of Salta province (Law 7543) with overlap on Category II (yellow) of the UAH Closed Shrubland Fine Textural Class/Thick and Open Bush/Bare areas in regularly flooded sectors (diagonal bars). The identified shrubs have low aptitude for sustainable use, which restricts the 'yellow' usable area. The rest of this area - with shelter from the edges of streams - would correspond to the UAH Closed Forest and Open Forest middle textural class, in which the expectations of sustainability are higher.

Sustentabilidad, que se refiere a la necesidad de evaluar la actitud de cada sector para ofrecer sustentabilidad a largo plazo antes de efectuar actividades para el cambio de uso del suelo.

Si se superpone al mapa del OTBN las áreas cubiertas con arbustos (Figura 3), se estima una superficie 56131 ha $(30 \%)$ dentro del sector zonificado amarillo del SPChSA, cuya categorización se debería revisar. A su vez, tendrían que surgir nuevos enfoques para abordar el ordenamiento ambiental del territorio. Esta revisión se debería enfocar en las importantes funciones ecosistémicas que poseen los estratos arbustivos en estos sectores. Los arbustos son vitales en el funcionamiento de los ecosistemas semiáridos, ya que confieren estabilidad estructural y ayudan a mantener su integridad al evitar o aminorar la erosión de los suelos. Además, contribuyen de manera significativa a mantener una biodiversidad elevada (Gutiérrez and Squeo 2004). Al contrario de la creencia generalizada de que una mayor presencia de arbustos se asocia a ecosistemas degradados, Soliveres et al. (2014) demostraron que una cobertura intermedia de esta vegetación leñosa genera ecosistemas más 
diversos y suelos más fértiles, por lo que los planes de manejo o conservación deberían tender a mantener o alcanzar esa cobertura.

En el caso del SPChSA, un análisis particular ameritan los Arbustales Abiertos en áreas desnudas en sectores regularmente anegados ubicados en la porción sur del territorio, ya que corresponden a los sectores identificados por Firpo Lacoste (2018) como Terrazas Fluviales. Estos sectores constituyen el tope de una secuencia fluvial que es alcanzada por los pulsos actuales del Bermejo. Forman parte de una extensa red de humedales emergentes, arbustivos y forestales que constituyen hábitats destacados, de alta importancia biológica, dado que poseen influencia a lo largo de kilómetros. Allí viven decenas de familias aborígenes y criollas que aprovechan múltiples bienes y servicios ecosistémicos para su reproducción social (Córdoba 2018).

Los resultados aquí presentados alertan sobre la importancia de identificar, mapear y dar a conocer las UAH del territorio. En el análisis de las UAH no deberían estar ausentes los vínculos que existen con los complejos sistemas socioproductivos locales, como insumo para ordenar el uso del suelo con reales perspectivas de sustentabilidad.

Agradecimientos. Agradecemos el apoyo recibido del Observatorio Nacional de Degradación de Tierras y Desertificación y del Ministerio de Ambiente y Desarrollo Sustentable de la Nación, Proyecto Soporte de decisiones para la integración y ampliación del Manejo Sustentable de Tierras GCP/GLO/ 337/GFF.

\section{REFERENCIAS}

Alencar da Silva, A., K. M. Parodi D’avila, M. C. Silva Nóbrega, and R. Opazo Albarrán. 2019. Variabilidad espacial y temporal de la cobertura vegetal de los años 1984 a 2011 en la cuenca hidrográfica del río Moxotó, Pernambuco, Brasil. Diálogo Andino 58:139-150. https://doi.org/10.4067/S0719-26812019000100139.

Araya Morales, E. 2009. Manual de procesos en fotografías aéreas e Imágenes de satélite. Documento técnico № 11 Área: Evaluación de Tierras. Proyecto Microcuenca Plantón - Pacayas San José, Costa Rica.

Bouyoucos, G. J. 1962. Hydrometer method for making particle size analysis de soils. Agronomy Journal 54:464-465. https://doi.org/10.2134/agronj1962.00021962005400050028x.

Cabral, C. M., M. Negri, E. Raineri, and V. Geipel. 2009. Sistema de Información Geográfico de la provincia de Salta. Planificación, gestión, monitoreo y control del desarrollo sustentable en bosques nativos. Informe Final Ministerio de Desarrollo Económico. Consejo Federal de Inversiones, Salta, Argentina.

Cabrera, A. L. 1976. Regiones Fitogeográficas Argentinas. En Enciclopedia Argentina de Agricultura y Jardinería. Tomo II. Segunda Edición. Ed: ACME S.A.C.I. Buenos Aires, Argentina.

Camardelli, M. C., M. Barbera, P. Colina, and S. Reyes. 2015. Tecnologías apropiadas en predios de ganaderos criollos chaqueños: el espacio tecnológico (ET) como herramienta de construcción. Pp. 169-196 en S. Ataides (eds.). Desarrollo Rural en Debate. Estudios en el espacio agrario salteño. La Colmena, Argentina.

Camardelli, M. C., V. H. Caruso, and S. Miranda. 2012. Competencia entre pasturas implantadas bajo el bosque y renovales de especies nativas arbóreas y arbustivas en el chaco salteño. Argentina. In: $2^{\circ}$ Congreso Nacional de Sistemas Silvo-pastoriles: actas/edición literaria. 1ª edición. Pp. 248-253. Santiago del Estero: Ediciones INTA.

Camardelli, M. C., V. H. Caruso, S. M. Pérez de Bianchi, D. Pérez, and S. Miranda. 2005. Evaluación de cambios tempranos en la calidad de los suelos relacionados con el carbono en tierras ganaderas del chaco semiárido salteño. Revista Científica Agropecuaria 9:173-179.

Caruso, V. H., M. C. Camardelli, and S. Miranda. 2012. Efecto del método de desmonte sobre los indicadores de calidad del suelo y la condición de las pasturas en el Chaco Semiárido Salteño. AGRISCIENTIA XXIX:99-105.

Chuvieco, E. 1995. Fundamentos de teledetección espacial, 2aㅡ edición. Ed. Rialp S.A. Madrid, España.

Córdoba, G. 2018. Servicios Ecosistémicos del Chaco Semiárido de Salta. Temas de Biología y Geología del NOA. Revista de Divulgación Científica del Instituto de Bio y Geociencias 8(2):30-35.

Despósito, C. D., and T. Ledesma. 2015. Pautas preliminares y teóricas para el aprovechamiento silvopastoril en bosques nativos de dos quebrachos de Salta. En $3^{\circ}$ Congreso Nacional de Sistemas Silvo-pastoriles (340-344). Santiago del Estero: Ediciones INTA.

Dorta, J. C., M. Gimeno Ortiz, and A. R. Ojeda. 2014. Unidades Ambientales Homogéneas como herramienta para la ordenación territorial y la caracterización de litorales áridos. Vegueta. Anuario de la Facultad de Geografía e Historia 14:199-228.

FAO. 2020. Evaluación de los Recursos Forestales Mundiales (FRA). Documento de Trabajo No. 188. FAO, Roma. Italia. Pp. 33.

Firpo Lacoste, F. 2018. Inventario de Humedales Salto-Chaqueños. En El Agua Subterránea: Recurso sin Fronteras: Humedales Vinculadas al Agua Subterránea. Rodolfo Fernando García [et al.]. Primera Edición. Editorial de la Universidad Nacional de Salta. Salta. Pp. 197-204.

Gaitán, J. J., D. Bran, G. Oliva, G. Ciari, V. Nakamatsu, J. Salomone, D. Ferrante, G. Buono, V. Massara, G. Humano, D. Celdrán, W. Opazo, and F. T. Maestre. 2013. Evaluating the performance of multiple remote sensing indices to predict the spatial variability of ecosystem structure and functioning in Patagonian steppes. Ecological Indicators 
34:181-191. https://doi.org/10.1016/j.ecolind.2013.05.007.

García, R. M. 2004. Inferencia estadística y diseño de experimentos. Ed. Eudeba, Buenos Aires, Argentina.

Guerschman, J. P., J. M. Paruelo, C. Di Bella, M. C. Giallorenzi, and F. Pacin. 2003. Land cover classification in the Argentine Pampas using multi-temporal Landsat TM data, International Journal of Remote Sensing 24:3381-3402. https://doi.org/10.1080/0143116021000021288

Guevara, A., S. A. Castrillo, I. Garzarón, E. J. Condorí, C. V. Giordano, and M. C. Camardelli. 2020. Características del estrato arbustivo en dos sistemas de manejo de bosque para la ganadería en el Chaco semiárido salteño. Tercer Congreso Internacional del Gran Chaco americano: Territorio e Innovación. Santiago del Estero, Argentina. UNSE.

Gutiérrez, J. R, and F. A. Squeo. 2004. Importancia de los arbustos en los ecosistemas semiáridos de Chile. Ecosistemas 13:36-45.

Mas, J., J. Reyes Díaz-Gallegos, and A. Pérez Vega. 2003. Evaluación de la confiabilidad temática de mapas o de imágenes clasificadas: una revisión. Investigaciones Geográficas, Boletín del Instituto de Geografía, UNAM 51:53-72.

Morello, J., S. Matteucci, A. Rodríguez, and M. Silva. 2012. Ecorregiones y complejos ecosistémicos argentinos. Ed: Orientación Gráfica Editora.

Schmidt, M. 2014. Bosques nativos en Salta: Entre el ordenamiento y los re-(des)ordenamientos posibles. Geograficando 10:1-20.

Soliveres, S., F. Maestre, D. Eldridge, M. Delgado-Baquerizo, J. L. Quero, M. Bowker, and A. Gallardo. 2014. Plant diversity and ecosystem multifunctionality peak at intermediate levels of woody cover in global drylands. Global Ecology Biogeography Glob Ecol Biogeogr 23(12):1408-1416. https://doi.org/10.1111/geb.12215. PMID: 25914607. https://doi.org/10.1111/geb.12215.

Therburg, A., M. L Corso, M. Stamati, C. Bottero, P. Lizana, and V. Pietragalla. 2019. Informe Síntesis de Resultados de la Evaluación de la Degradación de Tierras: 2012-2017. Observatorio Nacional de la Degradación de Tierras y Desertificación. SAyDS, CONICET, IADIZA-Conicet, INTA, FAUBA, Buenos Aires, Argentina.

Volante, J. N., and A. R. Bianchi. 2003. Índice Verde: Índice de Vegetación Normalizado Diferencial (NDVI) de las Yungas y del Chaco Semiárido en el Noroeste Argentino. Sitio Argentino de Producción Animal.

Volante, J. N. (coord.), A. Collado, E. B. Ferreyra, C. López, M. Navarro, A. Pezzola, and M. I. Puentes. 2009. Informe Técnico Unificado PNECO 1643. Monitoreo de la Cobertura y el Uso del Suelo a partir de sensores remotos. Programa Nacional de Ecorregiones, INTA, Buenos Aires, Argentina. 